\title{
Strong Proximity Josephson Coupling in Vertically Stacked NbSe2-Graphene-NbSe2 van der Waals Junctions
}

DOI:

10.1021/acs.nanolett.7b02707

\section{Document Version}

Accepted author manuscript

Link to publication record in Manchester Research Explorer

\section{Citation for published version (APA):}

Kim, M., Park, G. H., Lee, J., Lee, J. H., Park, J., Lee, H., Lee, G. H., \& Lee, H. J. (2017). Strong Proximity Josephson Coupling in Vertically Stacked NbSe ${ }_{2}$-Graphene-NbSe ${ }_{2}$ van der Waals Junctions. Nano Letters, 17(10), 6125-6130. https://doi.org/10.1021/acs.ranalett.7b02707

\section{Published in:}

Nano Letters

\section{Citing this paper}

Please note that where the full-text provided on Manchester Research Explorer is the Author Accepted Manuscript or Proof version this may differ from the final Published version. If citing, it is advised that you check and use the publisher's definitive version.

\section{General rights}

Copyright and moral rights for the publications made accessible in the Research Explorer are retained by the authors and/or other copyright owners and it is a condition of accessing publications that users recognise and abide by the legal requirements associated with these rights.

\section{Takedown policy}

If you believe that this document breaches copyright please refer to the University of Manchester's Takedown Procedures [http://man.ac.uk/04Y6Bo] or contact uml.scholarlycommunications@manchester.ac.uk providing relevant details, so we can investigate your claim.

\section{OPEN ACCESS}




\section{Strong proximity Josephson coupling in vertically}

\section{stacked $\mathrm{NbSe}_{2}$-graphene-NbSe 2 van der Waals}

\section{junctions}

Minsoo $\mathrm{Kim}^{\dagger}$, Geon-Hyoung Park, Jongyoon Yi, Jae Hyeong Lee, Jinho Park, Hyunwoo Lee, Gil-Ho Lee, and Hu-Jong Lee*

Department of Physics, Pohang University of Science and Technology, Pohang 37673, Republic of Korea

KEYWORDS: Graphene, $\mathrm{NbSe}_{2}$, two-dimensional materials, heterostructure, Josephson junction

\footnotetext{
ABSTRACT A layered two-dimensional superconducting material $2 \mathrm{H}-\mathrm{NbSe}_{2}$ is used to build a van der Waals heterostructure, where a proximity-coupled superconducting order can be induced in the interfacing materials. Vertically stacked $\mathrm{NbSe}_{2}-$ graphene- $\mathrm{NbSe}_{2}$ is fabricated using van der Waals interlayer coupling, producing defect-free contacts with a high interfacial transparency. The atomically thin graphene layer allows the formation of a highly coherent proximity Josephson coupling between the two $\mathrm{NbSe}_{2}$ flakes. The temperature dependence of the junction critical current $\left(I_{\mathrm{c}}\right)$ reveals short and ballistic Josephson coupling characteristics that agree with theoretical prediction. The strong Josephson coupling is confirmed by a large junction critical current density of $1.6 \times 10^{4} \mathrm{~A} / \mathrm{cm}^{2}$, multiple Andreev reflections in the subgap structure of the differential conductance, and a magnetic-field modulation of $I_{\mathrm{c}}$. This is the first demonstration of strongly proximity-coupled Josephson
} 
junctions with extremely clean interfaces in a dry-transfer-stacked van der Waals heterostructure.

The conducting characteristics of van der Waals stacked two-dimensional (2D) materials vary widely to be metallic, semiconducting, and insulating, where the unique 2D nature of these materials is investigated by isolating thin layers from the bulk material. ${ }^{1-3}$ The exotic properties of isolated 2D materials have generated a great deal of interest, and stacking the different 2D materials into van der Waals heterostructures provides an even more exciting platform to tune their electronic properties with enhanced material functionality. ${ }^{4,5}$ A range of phenomena may occur at the atomically flat interface of a heterostructure, ${ }^{6}$ including charge transfer, ${ }^{7}$ modification of the electronic band structure, ${ }^{8-10}$ and proximity effects. ${ }^{11,12}$ Among the assortment of $2 \mathrm{D}$ materials, $2 \mathrm{H}-\mathrm{NbSe}_{2}$ demonstrates robust superconductivity down to the monolayer limit with varying critical temperatures. ${ }^{13-16}$ Thus, within the heterostructure of $\mathrm{NbSe}_{2}$ and a clean interfaced 2D conductor, $\mathrm{NbSe}_{2}$ can induce a superconducting order in the contacting normal-state 2D conductor. ${ }^{17,18}$

In this study, strongly proximity-coupled Josephson junctions are formed ${ }^{19}$ in the heterostructure of monolayer graphene, an atomically thin carbon layer of honeycomb lattice structure, vertically sandwiched between two $\mathrm{NbSe}_{2}$ layers. Each layer was sequentially drytransferred onto the other to form the stack. The superconducting pair current flows through the graphene layer, satisfying the Josephson relations, ${ }^{19-21}$ where the magnitude of the supercurrent depends on the phase difference of the superconducting order between the two $\mathrm{NbSe}_{2}$ layers. The proximity-type Josephson coupling observed in our dry-transfer-stacked $\mathrm{NbSe}_{2}$-graphene- $\mathrm{NbSe}_{2}$ heterostructure devices is in stark contrast to the tunneling-type junction behavior illustrated previously in directly stacked $\mathrm{NbSe}_{2}-\mathrm{NbSe}_{2}$ junctions. ${ }^{22}$ Using single-atom-thick graphene between the superconducting layers leads to ballistic transport in the thickness direction without carrier scattering (channel length $l<$ mean free path $l_{\mathrm{mfp}}$ ), while maintaining a strong superconducting phase coherence $(l<$ superconducting phase coherence length $\xi)$. These conditions produce short $(l<\xi)$ and ballistic $\left(l<l_{\mathrm{mfp}}\right)$ Josephson 
coupling in the $\mathrm{NbSe}_{2}-$ graphene- $\mathrm{NbSe}_{2}$ heterostructure with the coupling much stronger than our previous report on short and ballistic Josephson coupling in another type of vertical graphene junctions. ${ }^{23}$ The strong Josephson coupling arises from the direct contact between $\mathrm{NbSe}_{2}$ and graphene. The Andreev bound state forms in the graphene layer, which acts as an atomically thin normal-conducting spacer with clean and flat interfaces for both $\mathrm{NbSe}_{2}$ layers. As a single Andreev bound state mediates supercurrent flow in short and ballistic Josephson junctions, ${ }^{24}$ vertically stacking the $\mathrm{NbSe}_{2}-$ graphene-NbSe 2 van der Waals heterostructure by dry-transfer techniques offers a useful way of realizing various superconducting hybrid quantum devices. ${ }^{25-27}$

(a)

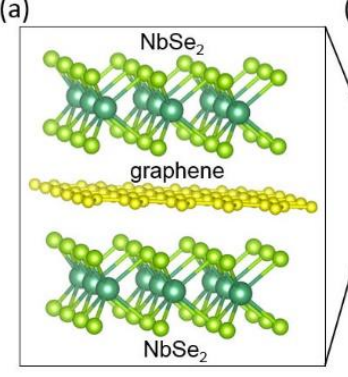

(b)

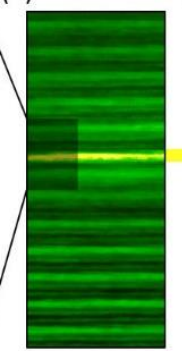

(c)

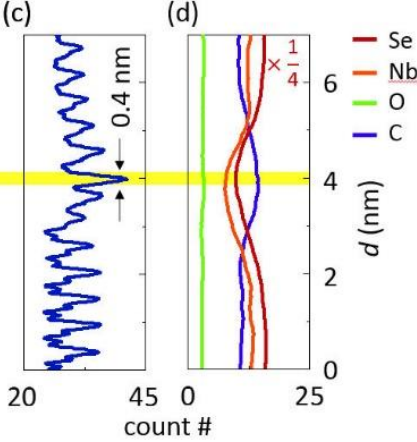

(e)

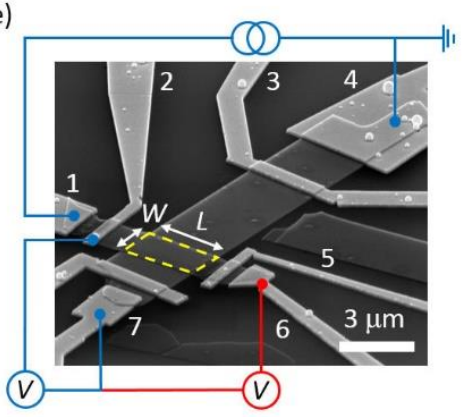

Figure 1. (a) Schematic of the atomic structure of the $\mathrm{NbSe}_{2}-$ graphene- $-\mathrm{NbSe}_{2}$ heterostructure. (b) Scanning transmission electron microscope (STEM) image of the junction vertical cross-section. (c) A slice profile of the STEM image and (d) corresponding energy dispersive spectroscopy data. (e) Scanning electron microscope image of the device with the three-terminal $\left(I_{1-4}-V_{2-7}\right)$ or four-terminal $\left(I_{1-4}-V_{6-7}\right) I-V$ measurement configuration. The boundary of the graphene layer encapsulated between $\mathrm{NbSe}_{2}$ crystals is denoted by dashed line. The magnetic field was applied in the planar direction, along the width $(W)$ of the junction.

$\mathrm{NbSe}_{2}-$ graphene- $\mathrm{NbSe}_{2}$ junctions (Fig. 1a) were prepared by dry processing, which produces a clean interface via van der Waals coupling. ${ }^{28}$ Each layer was sequentially drytransferred under ambient conditions. The Josephson coupling characteristics were investigated below the superconducting critical temperature of the $\mathrm{NbSe}_{2}$ crystals, $T_{\mathrm{c}}=6.55$ 
K. At a base temperature $T$ of $0.2 \mathrm{~K}$, a large junction critical current density $\left(J_{\mathrm{c}}\right)$ of $1.6 \times$ $10^{4} \mathrm{~A} / \mathrm{cm}^{2}$ was obtained with a small junction resistance $\left(<5 \Omega / \mu \mathrm{m}^{2}\right)$, indicating clean heterostructure interfaces. Multiple Andreev reflection (MAR) features in the subgap structure of differential conductance also supported the transparent transport via the graphene layer. Although the measured $I_{\mathrm{c}} R_{\mathrm{n}}$ product falls short of the ideal value, the observed temperature dependence of the junction critical current $\left(I_{\mathrm{c}}\right)$ provides further evidence of the highly transparent Josephson coupling characteristics that agree with theoretical predictions for short and ballistic Josephson coupling.

Figure $1 \mathrm{~b}$ shows a scanning transmission electron microscope (STEM) image of the vertical cross-section of the junction, where the graphene layer is highlighted in yellow. The STEM image and the slice plot in Fig. 1c verify that clean and flat interfaces were obtained between the graphene and $\mathrm{NbSe}_{2}$ layers. The thickness of the graphene layer was measured to be $\sim 0.4 \mathrm{~nm}$, consistent with the thickness of a single graphene layer. Subsequent energy dispersive spectroscopy (EDS) of the cross-section (Fig. 1d) confirmed that no oxidation took place at the interface of the $\mathrm{NbSe}_{2}$ and graphene. Oxidation of $\mathrm{NbSe}_{2}$ is considered as a factor that degrades the junction characteristics with the reduced junction transparency ${ }^{16,22}$ (see Method and Supplementary Information regarding the device fabrication process and measurements on the junction with oxidized interfaces).

Figure 1e is a scanning electron microscope (SEM) image of the vertically stacked device. The graphene (dashed line) was inserted between two $\mathrm{NbSe}_{2}$ crystals, where the cross-sectional dimension $W \times L$ was $2 \times 3.5 \mu \mathrm{m}^{2}$, as measured by SEM. Electrodes $1,2,5$, and $6(3,4$, and 7$)$ were contacted to the top (bottom) $\mathrm{NbSe}_{2}$ crystal layer. The thickness of the top (bottom) $\mathrm{NbSe}_{2}$ crystal was about $40 \mathrm{~nm}(80 \mathrm{~nm})$, as measured by atomic force microscopy. A bias current was passed through the graphene between electrodes 1 and 4, while the potential difference across the junction was monitored at electrodes 2 and 7, and 6 and 7 for the three- and four-probe measurement configuration, respectively. Standard lock-in techniques were used to measure the junction conduction characteristics using a dc bias 
current, modulated with $100 \mathrm{nA}$ at a frequency of $13.3 \mathrm{~Hz}$ in a dilution fridge (Oxford Model Kelvinox) at a base $T$ of $0.2 \mathrm{~K}$.
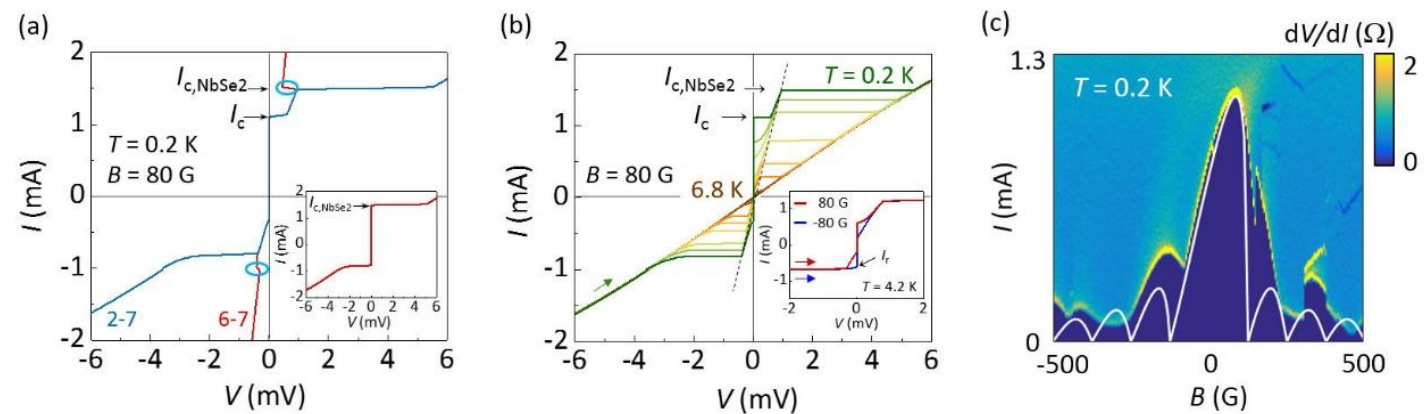

Figure 2. (a) Current-voltage (I-V) characteristics of an $\mathrm{NbSe}_{2}-$ graphene- $\mathrm{NbSe}_{2}$ junction, taken in three-terminal $\left(I_{1-4^{-}} V_{2-7} ;\right.$ blue curve $)$ and four-terminal $\left(I_{1-4^{-}} V_{6-7} ;\right.$ red curve $)$ configurations. Two transitions in each $I-V$ curve correspond to the critical current of the Josephson junction $\left(I_{\mathrm{c}}\right)$ and the top $\mathrm{NbSe}_{2}$ crystalline electrode $\left(I_{\mathrm{c}}{ }^{\text {top }}{ }_{\text {NbSe2}}\right)$. Inset: $I-V$ curves of the top $\mathrm{NbSe}_{2}$ crystalline electrodes, measured in $I_{1-4}-V_{2-5}$ configuration. (b) $I-V$ curves taken in the three-terminal configuration for temperature $T=0.2,2.7,4.3,5.8,6.1,6.3,6.6$ and 6.8 $\mathrm{K}$, and magnetic field $B=80 \mathrm{G}$. The slope of the dashed line corresponds to the junction resistance. Inset: $I-V$ curves measured at $T=4.2 \mathrm{~K}, B=80 \mathrm{G}$ (red) and $-80 \mathrm{G}$ (blue). $I_{\mathrm{r}}$ represents the retrapping current of the junction. Colored arrows indicate the direction of the current bias. (c) Fraunhofer pattern of $I_{\mathrm{c}}$, where the white curve is a theoretical fit to the experimental data, taking the self-field effect into consideration.

$\mathrm{NbSe}_{2}$ becomes superconducting below $T=6.55 \mathrm{~K}$ and the Josephson supercurrent occurs slightly below the temperature of $T_{\mathrm{c}}=6.50 \mathrm{~K}$. Figures $2 \mathrm{a}$ and $2 \mathrm{~b}$ show the forward current biasing current-voltage $(I-V)$ characteristics of the junction at a range of $T$ from $0.2 \mathrm{~K}$ to $6.8 \mathrm{~K}$ and an in-plane magnetic field $B$ of $80 \mathrm{G}$, with two specific transitions denoted by arrows. The low-bias transition occurred at the critical current $I_{\mathrm{c}}(=1.11 \mathrm{~mA})$ of the Josephson junction at a base $T$ of $0.2 \mathrm{~K}$, corresponding to a junction critical current density $J_{\mathrm{c}}$ 
of $\sim 1.6 \times 10^{4} \mathrm{~A} / \mathrm{cm}^{2}$. As explained below, an $80 \mathrm{G}$ in-plane field gives a maximum value of $I_{\mathrm{c}}$ in the presence of a large self-field. The high-bias transition corresponds to the critical current of the top $\mathrm{NbSe}_{2}$ crystalline electrode, $I_{\mathrm{c}}{ }^{\text {top }}{ }_{\text {,NbSe}}(=1.50 \mathrm{~mA}$; inset of Fig. 2a). The critical current of the bottom $\mathrm{NbSe}_{2}$ crystalline electrode $\left(I_{\mathrm{c}}{ }^{\text {bottom }}{ }_{, \mathrm{NbSe} 2}=2.31 \mathrm{~mA}\right)$ is beyond the plotting range of Figs. $2 \mathrm{a}$ and $2 \mathrm{~b}$. The disparity of the critical current between the top and bottom $\mathrm{NbSe}_{2}$ electrodes arose from the difference in each of the cross-sectional areas. Even in the three-terminal measurement configuration $\left(I_{1-5}-V_{2-3}\right)$ adopted to measure $I_{\mathrm{c}}{ }^{\text {top }}{ }_{\text {NbSe2 } 2}$, the lead resistance was not included in the measured junction resistance, as the $\mathrm{NbSe}_{2}$ leads were superconducting for $I<I_{\mathrm{c}}{ }^{\text {top }}{ }_{\text {NbSe2 }}$. Thus, the $I-V$ curves for three- and four-terminal measurements (blue and red curves in Fig. 2a, respectively) follow each other closely for $I<$ $I_{\mathrm{c}}{ }^{\text {top }}{ }_{\text {,NbSe2}}$. The large voltage jump at $I_{\mathrm{c}}{ }^{\text {top }}{ }_{\text {,NbSe2 }}$ with increasing bias arises from the in-plane addendum resistance across the region $w \times a$ in Fig. $\mathrm{S} 1 \mathrm{i}$ as the top $\mathrm{NbSe}_{2}$ layer becomes normal-conducting. For $I_{\mathrm{c}}{ }^{\text {top }}{ }_{\text {NbSe2 } 2}<I$, the current flow in the junction area becomes nonuniform as the square resistance of a $\mathrm{NbSe}_{2}$ electrode (the top electrode in this case) in its normal conducting state becomes comparable to or larger than the junction resistance corresponding to the uniform current flow (say, for $I_{\mathrm{c}}<I<I_{\mathrm{c}}{ }^{\text {top }}{ }_{\text {,NbSe} 2}$ in this device). This would lead to an anomalous drop of the junction resistance in the cross-junction measurement configuration, causing the small backward resistance-jump anomaly denoted by void cyan circles in the four-terminal (red) measurement curve (refer to Figs. S2 and S3 for more details). It is assumed that the same non-uniform current distribution was present in the threeterminal (blue) curve, but it was masked by inclusion of the large in-plane addendum resistance in the top $\mathrm{NbSe}_{2}$ electrode.

Figure $2 \mathrm{~b}$ shows the $I-V$ curves taken in the three-terminal configuration for temperatures ranging from 0.2 to $6.8 \mathrm{~K}$ in a magnetic field $(B)$ of $80 \mathrm{G}$. The slope of the $I-V$ curves (emphasized by the dotted line) corresponds to the normal-state junction resistance $R_{\mathrm{n}}$ $(\sim 0.59 \Omega)$. In general, $R_{\mathrm{n}}$ of a junction is estimated from the slope $d V / d I$ for a bias that is sufficiently above the junction critical current $I_{\mathrm{c}}$. In this study, however, only a small difference was observed between the junction critical current $I_{\mathrm{c}}$ and $I_{\mathrm{c}}{ }^{\text {top }}{ }_{\text {,NbSe2}}$, which made it 
difficult to estimate the junction resistance accurately. Junction resistance based on an incompletely developed normal-junction state leads to an underestimation of the value $R_{\mathrm{n}}$.

The inset of Fig. $2 \mathrm{~b}$ shows the $I-V$ characteristic curves, measured at $T=4.2 \mathrm{~K}$ for $B=$ $80 \mathrm{G}$ (red) and $-80 \mathrm{G}$ (blue). The peculiar result that the retrapping current $I_{\mathrm{r}}$ was larger than $I_{\mathrm{c}}$ at $B=-80 \mathrm{G}$ was due to the self-field effect arising from the large $J_{\mathrm{c}}$ of the junction. ${ }^{29-32}$ When a large current flows between the superconducting electrodes of the junction, the dimensions of which $(L, W)$ are comparable to the Josephson penetration depth $\lambda_{\mathrm{J}}$, the large junction current induces a significant in-plane magnetic field. This results in a skewness of maximum peaks of $I_{\mathrm{c}}$ and $I_{\mathrm{r}}$ from those at zero self-field (see Fig. $2 \mathrm{c}$ ). $\lambda_{\mathrm{J}}$ $\left(=\sqrt{h / 4 \pi \mu_{0} e(l+2 \lambda) J_{c}}\right) \quad$ of the Josephson junction is estimated to be about $5.7 \mu \mathrm{m}$ at base temperature, where $h$ is Planck's constant, $\mu_{0}$ is the vacuum permeability, $e$ is the electron charge, and $\lambda$ is the London penetration depth of $\mathrm{NbSe}_{2}$ crystals (see the following analysis for the $B$-field modulation of the critical current). The maximum peaks of $I_{\mathrm{c}}$ and $I_{\mathrm{r}}$ (not shown) of the principal lobe occur at $B=80 \mathrm{G}$ and $-80 \mathrm{G}$, respectively. Thus, a proper comparison of $I_{\mathrm{c}}$ and $I_{\mathrm{r}}$ of the junction should be done for the values of $I_{\mathrm{c}}$ at $B=80 \mathrm{G}$ and $I_{\mathrm{r}}$ at $-80 \mathrm{G}$. As shown in the inset of Fig. $2 \mathrm{~b}$, both $I_{\mathrm{c}}$ at $B=80 \mathrm{G}$ and $I_{\mathrm{r}}$ at $B=-80 \mathrm{G}$ are found to be $0.60 \mathrm{~mA}$. From the resistively and capacitively shunted junction (RCSJ) model, the negligible difference between $I_{\mathrm{c}}$ and $I_{\mathrm{r}}$ indicates that the junction was in the overdamped limit of a proximity Josephson junction. ${ }^{33}$

The self-field effect becomes more conspicuous in the results for the $B$-field dependence of the $I-V$ characteristics, shown in Fig. 2c. It was manifested as the skewed modulation of the junction critical current with $B$ field. We note that the skewed modulation in the Fraunhofer pattern was not caused by the flux trapping during the measurements but by the self-field effect. The Fraunhofer pattern forms symmetrically with respect to the origin ( $B$ $=0$ and $I=0$ ) upon simultaneous reversal of $I$ and $B$, which also appears in other devices (see Fig. S4 and S6). The skew of $I_{\mathrm{c}}$ modulation with $B$ field was quantitatively analyzed with an 
approximation of uniform current distribution in the junction. The Fraunhofer critical-current modulation, as a function of in-plane $B$, was then modified as

$$
\frac{I\left(\Phi / \Phi_{0}\right)}{I_{0}}=\left|\frac{\sin \pi\left(\Phi / \Phi_{0}+a I\left(\Phi / \Phi_{0}\right) / I_{0}\right)}{\pi\left(\Phi / \Phi_{0}+a I\left(\Phi / \Phi_{0}\right) / I_{0}\right)}\right|,
$$

where $\Phi_{0}(=h / 2 e)$ is the magnetic flux quantum, $\Phi[=B L(l+2 \lambda)]$ is the magnetic flux threading the junction area, and $a\left(=L^{2} / 4 \pi \lambda_{\mathrm{J}}^{2}\right)$ is a parameter representing the strength of the self-field effect. ${ }^{31}$ The white curve in Fig. $2 \mathrm{c}$ shows the best fit to the data following Eq. (1). Periodic modulation of $I_{\mathrm{c}}$ as a function of $B$ field at a period of $128 \mathrm{G}$ corresponds to the junction geometry with an estimation of $2 \lambda \sim 50 \mathrm{~nm}$. The non-monotonic suppression of the $I_{\mathrm{c}}$ maximum at each lobe and finite $I_{\mathrm{c}}$ at $\Phi=n \Phi_{0}(n= \pm 1, \pm 2, \ldots)$ are possibly due to the self-field effect modifying the actual current distribution across the junction depending on its geometry, ${ }^{29-31}$ which is not considered in Eq. (1). The abrupt jumps of $I_{\mathrm{c}}$ at a few finite values of $B$ field are attributed to a sudden penetration of Abrikosov magnetic vortices into the typeII superconductor $\mathrm{NbSe}_{2}$, owing to slight misalignment of the applied field from the planar direction.
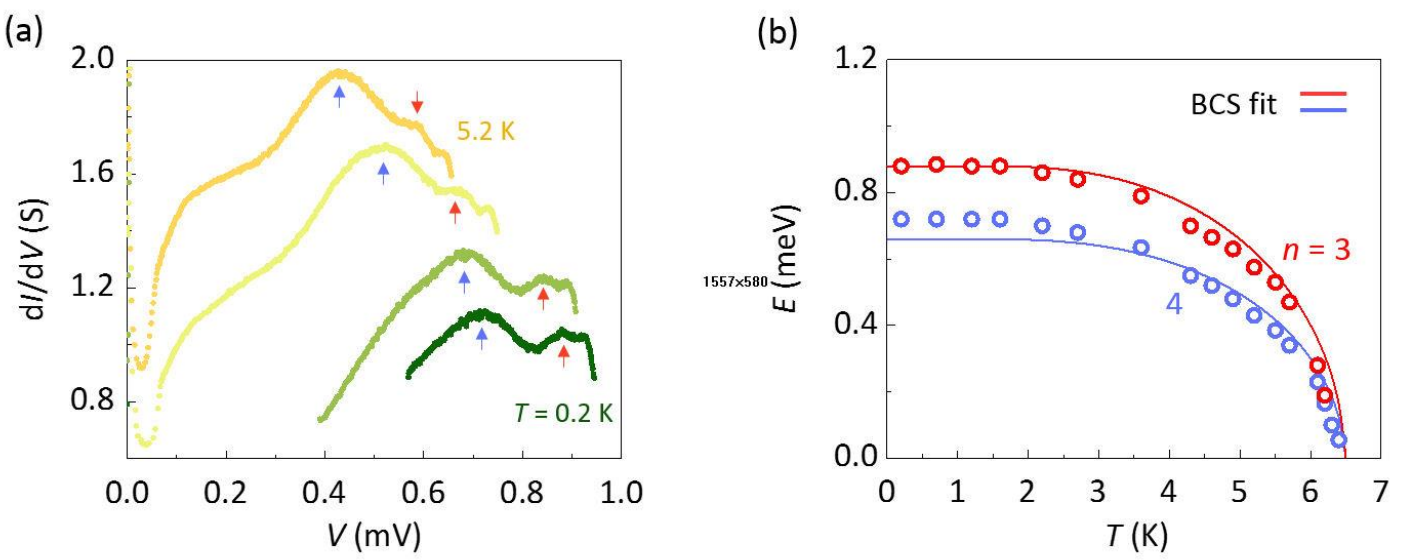

Figure 3. (a) Multiple Andreev reflection (MAR) in the differential conductance $d I / d V$ curve measured at temperatures $T$ of $0.2,2.7,4.6$, and $5.2 \mathrm{~K}$. Subgap conductance peaks are indicated by arrows. (b) Temperature dependence of subgap conductance peaks (symbol) 
satisfying the MAR relation for $n=3$ (red) and 4 (blue). Solid lines are the best fits to the Bardeen-Cooper-Schrieffer (BCS) temperature dependence for a superconducting energy gap.

The highly transparent nature of Josephson junctions can be further demonstrated by the MAR effect in the $I-V$ characteristics. Figure 3a shows the subgap structure of the differential conductance, caused by the MAR for a few representative temperatures at $T=0.2$, 2.7, 4.6, and $5.2 \mathrm{~K}$, where the subgap conductance peaks are indicated by arrows. The appearance of this subgap MAR feature, which is absent in the tunneling-type Josephson coupling, serves as the direct signature of the proximity-type Josephson coupling. ${ }^{34}$ For a highly transparent superconductor-normal-conductor-superconductor proximity junction, the MAR occurs at $V_{\mathrm{MAR}}=2 \Delta / n e$, where $n$ is an integer and $\Delta$ is the superconducting gap energy of the $\mathrm{NbSe}_{2}$ electrodes. As the temperature increases, the voltage of the MAR peaks or the value of $\Delta$ is gradually reduced. By fitting the temperature dependence (symbols) to the Bardeen-Cooper-Schrieffer (BCS) theory (solid lines) in Fig. 3b, we confirm that the peaks correspond to $n=3$ (red) and 4 (blue), satisfying the MAR condition. Again, owing to the small difference between $I_{\mathrm{c}}$ and $I_{\mathrm{c}}{ }^{\text {top }}{ }_{\text {NbSe2}}$, it was not possible to observe any conductance peaks for $n=1$ and 2 of the MAR. The abrupt decrease of the differential conductance for $V$, slightly above the MAR peak voltage $n=3$ (red arrows), is caused by the switching to the normal conducting state at $I_{\mathrm{c}}^{\text {top }}{ }_{\text {,NbSe} 2}$, where the top $\mathrm{NbSe}_{2}$ electrode loses its superconductivity (see Supplementary Information for more pronounced MAR peaks in another device). From the fit in Fig. 3b, the value of the gap $\Delta$ is estimated to be $1.32 \mathrm{meV}$, which is close to the known energy gap of crystalline $\mathrm{NbSe}_{2} .{ }^{35,36}$

The maximum gap value $\Delta$ estimated from the MAR indicates that the $I_{\mathrm{c}} R_{\mathrm{n}}$ product of our Josephson junction is comparable to $\sim \Delta / 2$. As mentioned above, the vertical Josephson junction satisfies the theoretical criteria for short and ballistic Josephson coupling $\left(l<l_{\mathrm{mfp}}, \xi\right)$. However, the measured $I_{\mathrm{c}} R_{\mathrm{n}}$ product of our junction falls significantly short of the theoretical 
boundary value of $\sim 2.1 \Delta$, between the short-ballistic and short-diffusive Josephson coupling. ${ }^{19,35}$ We may attribute it to a reduction of $I_{\mathrm{c}}$ due to instrumental noise during the measurements or a Fermi velocity mismatch at the interface between the $\mathrm{NbSe}_{2}$ and graphene. ${ }^{38}$

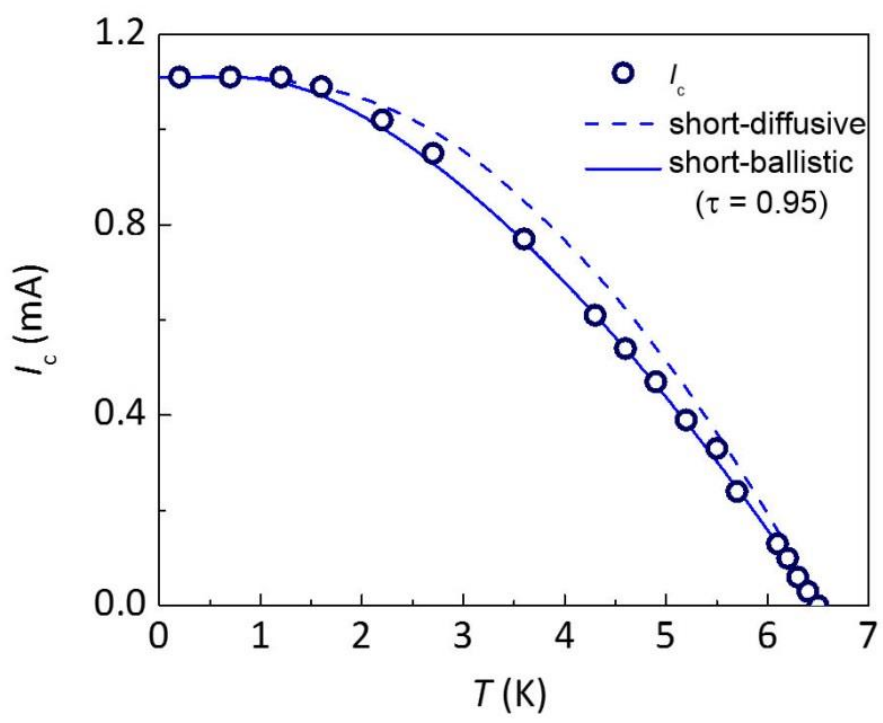

Figure 4. Temperature dependence of the junction critical current $I_{\mathrm{c}}$ (void symbols) in the $\mathrm{NbSe}_{2}-$ graphene- $\mathrm{NbSe}_{2}$ vertical Josephson junction. The solid (dashed) curve is the best fit corresponding to the short and ballistic (short and diffusive) Josephson coupling.

Figure 4 shows the temperature dependence of junction critical current $I_{\mathrm{c}}$ (void symbols) in the vertical Josephson junction. The atomically thin channel length leads to strong (short) Josephson coupling. ${ }^{23}$ The convex-shaped decrease of $I_{\mathrm{c}}$ with increasing temperature indeed suggests short Josephson coupling characteristics. To specify the junction type more precisely, i.e., short-diffusive or short-ballistic, the best fitting is carried out considering the current-phase relations of the short-diffusive and short-ballistic junction. The solid curve in Fig. 4 is the best fit obtained based on Eq. (2), which describes the currentphase relation of the short-ballistic Josephson junction ${ }^{37,39,40}$ 


$$
I_{J}(\phi, T)=\frac{e \Delta}{2 \hbar} \sum_{n=1}^{N} \frac{\tau_{n} \sin \phi}{\sqrt{1-\tau_{n} \sin ^{2}(\phi / 2)}} \tanh \left(\frac{\Delta}{2 k_{B} T} \sqrt{1-\tau_{n} \sin ^{2}(\phi / 2)}\right)
$$

where $\phi$ is the difference in macroscopic phase between two superconducting $\mathrm{NbSe}_{2}$ crystals and $\tau_{\mathrm{n}}$ is the transparency of the $n$-th conducting channel in graphene. The measured Josephson junction has many conducting channels over the junction area $\left(R_{\mathrm{Q}} / R_{\mathrm{n}}>10^{4}\right.$, where $R_{\mathrm{Q}}=h / e^{2}=25.8 \mathrm{k} \Omega$ is the quantum resistance). Thus, we describe $\tau_{\mathrm{n}}$ by the ensembleaveraged transparency $\tau$ for all conducting channels. Here, $\tau$ is best fit to $0.95( \pm 0.02)$, which is close to the full transparency limit of 1 . The value of $\tau$ verifies the highly transparent, short-ballistic Josephson coupling nature of the Josephson junction. As a reference, the best fit of the data to the short-diffusive Josephson coupling ${ }^{41}$ is given by the dashed curve in Fig. 4, which shows a degraded fit. This clearly confirms that the Josephson junction in this study represents short-ballistic Josephson coupling characteristics.

However, devices in the same $\mathrm{NbSe}_{2}-$ mono-layer graphene- $\mathrm{NbSe}_{2}$ structure but with oxidized interfaces and in a $\mathrm{NbSe}_{2}$-tri-layer graphene- $\mathrm{NbSe}_{2}$ structure without oxidized interfaces showed different $T$ dependence of $I_{\mathrm{c}}$. Although a convex-type decrease of $I_{\mathrm{c}}$ was observed with increasing $T$ in both cases, supporting the short Josephson junction characteristics, the detailed $T$ dependence of $I_{\mathrm{c}}$ was fitted equally well by both short-diffusive and short-ballistic models but with only low transparency (see Figs. S6 and S7). It indicates that the presence of a thin oxidized interface or a tri-layer graphene insert causes sufficient carrier scattering to make the transport via a junction diffusive. This study indicates that only the Josephson junction consisting of mono-layer graphene together with highly transparent $\mathrm{NbSe}_{2}$-graphene interfaces leads to the strong (short-ballistic) Josephson coupling.

The vertical $\mathrm{NbSe}_{2}-$ graphene- $\mathrm{NbSe}_{2}$ junctions in this study prepared by using drytransfer stacking shows significantly stronger Josephson coupling than another type of vertical graphene Josephson junction (Al-graphene-Al) we studied earlier, ${ }^{23}$ where a monolayer graphene was sandwiched between two electron-gun evaporated Ti/Al (8/200 nm 
thick) bilayers. The critical current $\left(I_{\mathrm{c}}=13.3 \mu \mathrm{A}\right)$ and the normal-state resistance $\left(R_{\mathrm{n}}=21.4\right.$ $\Omega$ ) of the Al-graphene-Al junction (lateral size; $\left.1.0 \times 6.3 \mu \mathrm{m}^{2}\right)$ is compared to those $\left(I_{\mathrm{c}}=1.11\right.$ $\mathrm{mA}$ and $R_{\mathrm{n}}=0.59 \Omega$ ) of the $\mathrm{NbSe}_{2}$-graphene- $\mathrm{NbSe}_{2}$ junction of this study with comparable lateral junction size $\left(2 \times 3.5 \mu \mathrm{m}^{2}\right)$. Thus, the Josephson coupling in an $\mathrm{NbSe}_{2}-$ graphene$\mathrm{NbSe}_{2}$ junction is stronger than in an Al-graphene- $\mathrm{Al}$ by almost two orders of magnitude. The weak coupling in the Al-graphene-Al junction was caused by the presence of the 8-nm-thick Ti layer, which formed a very tiny potential barrier $(\sim 170 \mathrm{meV})$ near the interface of $\mathrm{Ti}$ and graphene layers. Without the Ti layer no weak link formed across the monolayer graphene sheet and the Al-graphene-Al junction became a single superconducting layer. ${ }^{23}$ Thus, in an Al-graphene-Al junction, the formation of a weak link with proper coupling strength is fortuitous and tricky. In the case of the $\mathrm{NbSe}_{2}-\mathrm{NbSe}_{2}$ Josephson junction studied earlier, ${ }^{22}$ it was claimed that the coherent tunneling of Cooper pairs through a $\Gamma$-point of $\mathrm{NbSe}_{2}$ leads to the formation of a tunneling weak link. In comparison, in a dry-transfer stacked $\mathrm{NbSe}_{2}-$ graphene- $\mathrm{NbSe}_{2}$ junction in this study, a proximity-type weak link forms naturally with strong Josephson coupling strength by inserting normal-conducting mono-layer graphene sheet between $\mathrm{NbSe}_{2}$ layers. This scheme of preparing graphene Josephson junctions provides a higher versatility in applying such Josephson junctions to active quantum devices. The overlap of $\mathrm{K}$ points of mono-layer graphene and $\mathrm{NbSe}_{2}$ may have provided the strong proximity Josephson coupling in our device, although the difference in lattice constants and the uncertainty in the position of Fermi energy made it difficult to see any alignment effects between graphene and $\mathrm{NbSe}_{2}$ layer in this study.

In conclusion, we successfully prepared vertically stacked $\mathrm{NbSe}_{2}-$ graphene- $\mathrm{NbSe}_{2}$ heterostructures by the dry transfer of both $\mathrm{NbSe}_{2}$ and graphene layers. A clean interface with no potential barrier between $\mathrm{NbSe}_{2}$ and graphene produces a highly transparent, shortballistic proximity-type Josephson coupling $(\tau=0.95( \pm 0.02))$ with a large critical current density $J_{c}$. Constructing transparent Josephson junctions using $\mathrm{NbSe}_{2}$ and graphene represents an efficient and promising route to develop highly coherent hybrid systems and 
laterally scalable quantum device applications, such as Andreev-level qubits, based on 2D materials. The atomic vertical junction adopted in this study is also applicable to other newly emerging cleavable materials, opening an exciting route to explore exotic quantum phenomena at the atomic scale.

Heterostructures built using hexagonal lattice 2D materials have the potential for electronic band structure engineering through use of twisted angle alignment between the interfacing 2D materials. However, in this study, the lattice constant of $\operatorname{NbSe}_{2}\left(a_{\mathrm{NbSe} 2}=0.20\right.$ $\mathrm{nm})$ and graphene $\left(a_{\text {graphene }}=0.14 \mathrm{~nm}\right)$ were significantly different, making it hard to observe this effect. Using a transition metal dichalcogenide spacer layer with a similar lattice constant to $\mathrm{NbSe}_{2}$ could enable the observation of interesting electronic properties, including superlattice formation or Fermi surface matching.

\section{METHOD}

Device fabrication $\quad \mathrm{The}^{\mathrm{NbSe}} \mathrm{S}_{2}$ and graphene layers were sequentially dry-transferred to form the $\mathrm{NbSe}_{2}-$ graphene- $\mathrm{NbSe}_{2}$ heterostructure, which was placed onto a heavily electrondoped Si substrate capped with a 300-nm-thick oxidation layer (detailed transfer procedures are described in Supplementary Information). Then, electron beam lithography was used to form the contact regions. Ar-ion plasma etching in vacuum was used to expose a fresh $\mathrm{NbSe}_{2}$ interface immediately before the electron beam deposition of a Ti/Au $(5 \mathrm{~nm} / 120 \mathrm{~nm})$ bi-layer, which led to a low contact resistance between the $\mathrm{NbSe}_{2}$ and $\mathrm{Ti} / \mathrm{Au}$ contact electrodes. 


\section{AUTHOR INFORMATION}

\section{Corresponding Author}

*E-mail: hjlee@postech.ac.kr

\section{Present Addresses}

$\uparrow$ School of Physics and Astronomy, The University of Manchester, Manchester M13 9PL, UK.

\section{Author Contributions}

M. K. and H.-J. L. conceived the idea and designed the experiments. M. K. prepared the samples and performed the measurements. All authors analyzed the data. M. K. and H.-J. L. wrote the manuscript. G.-H. L. numerically analyzed the anomalous backward jump of the junction resistance. H.-J. L. supervised the study. All authors contributed to the discussion and approved the final version of the manuscript.

\section{Funding Sources}

This work was supported by the National Research Foundation (NRF) through the SRC Center for Topological Matter, POSTECH, Korea (Grant No. 2011-0030046) and the SRC Center for Quantum Coherence in Condensed Matter, KAIST, Korea (Grant No. 2016R1A5A1008184 for GHL).

\section{Notes}

The authors declare no competing financial interest 


\section{REFERENCES}

1. Wang, Q. H.; Kalantar-Zadeh, K.; Kis, A.; Coleman, J. N.; Strano, M. S. Nat. Nano. 2012, 7, (11), 699-712.

2. Xu, M.; Liang, T.; Shi, M.; Chen, H. Chem. Rev. 2013, 113, (5), 3766-3798.

3. Butler, S. Z.; Hollen, S. M.; Cao, L.; Cui, Y.; Gupta, J. A.; Gutiérrez, H. R.; Heinz, T. F.; Hong, S. S.; Huang, J.; Ismach, A. F.; Johnston-Halperin, E.; Kuno, M.; Plashnitsa, V. V.; Robinson, R. D.; Ruoff, R. S.; Salahuddin, S.; Shan, J.; Shi, L.; Spencer, M. G.; Terrones, M.; Windl, W.; Goldberger, J. E. ACS Nano 2013, 7, (4), 2898-2926.

4. Geim, A. K.; Grigorieva, I. V. Nature 2013, 499, (7459), 419-425.

5. Novoselov, K. S.; Mishchenko, A.; Carvalho, A.; Castro Neto, A. H. Science 2016, 353, (6298).

6. Haigh, S. J.; Gholinia, A.; Jalil, R.; Romani, S.; Britnell, L.; Elias, D. C.; Novoselov, K. S.; Ponomarenko, L. A.; Geim, A. K.; Gorbachev, R. Nat. Mater. 2012, 11, (9), 764-767.

7. Lee, C.-H.; Lee, G.-H.; van der Zande, A. M.; Chen, W.; Li, Y.; Han, M.; Cui, X.; Arefe, G.; Nuckolls, C.; Heinz, T. F.; Guo, J.; Hone, J.; Kim, P. Nat. Nano. 2014, 9, (9), 676-681.

8. Hunt, B.; Sanchez-Yamagishi, J. D.; Young, A. F.; Yankowitz, M.; LeRoy, B. J.;

Watanabe, K.; Taniguchi, T.; Moon, P.; Koshino, M.; Jarillo-Herrero, P.; Ashoori, R. C. Science 2013, 340, (6139), 1427-1430.

9. Dean, C. R.; Wang, L.; Maher, P.; Forsythe, C.; Ghahari, F.; Gao, Y.; Katoch, J.; Ishigami, M.; Moon, P.; Koshino, M.; Taniguchi, T.; Watanabe, K.; Shepard, K. L.; Hone, J.; Kim, P. Nature 2013, 497, (7451), 598-602.

10. Woods, C. R.; Britnell, L.; Eckmann, A.; Ma, R. S.; Lu, J. C.; Guo, H. M.; Lin, X.; Yu, G. L.; Cao, Y.; Gorbachev, R. V.; Kretinin, A. V.; Park, J.; Ponomarenko, L. A.; Katsnelson, M. I.; Gornostyrev, Y. N.; Watanabe, K.; Taniguchi, T.; Casiraghi, C.; Gao, H. J.; Geim, A. K.; Novoselov, K. S. Nat. Phys. 2014, 10, (6), 451-456.

11. Avsar, A.; Tan, J. Y.; Taychatanapat, T.; Balakrishnan, J.; Koon, G. K. W.; Yeo, Y.; Lahiri, J.; Carvalho, A.; Rodin, A. S.; O’Farrell, E. C. T.; Eda, G.; Castro Neto, A. H.; Özyilmaz, B. Nat. Commun. 2014, 5, 4875. 
12. Wang, Z.; Ki, D.-K.; Khoo, J. Y.; Mauro, D.; Berger, H.; Levitov, L. S.; Morpurgo, A. F. Phys. Rev. X 2016, 6, (4), 041020.

13. Frindt, R. F. Phys. Rev. Lett. 1972, 28, (5), 299-301.

14. Novoselov, K. S.; Jiang, D.; Schedin, F.; Booth, T. J.; Khotkevich, V. V.; Morozov, S. V.; Geim, A. K. Proc. Natl. Acad. Sci. U S A 2005, 102, (30), 10451-10453.

15. Staley, N. E.; Wu, J.; Eklund, P.; Liu, Y.; Li, L.; Xu, Z. Phys. Rev. B 2009, 80, (18), 184505.

16. Xi, X.; Wang, Z.; Zhao, W.; Park, J.-H.; Law, K. T.; Berger, H.; Forro, L.; Shan, J.; Mak, K. F. Nat. Phys. 2016, 12, (2), 139-143.

17. Efetov, D. K.; Wang, L.; Handschin, C.; Efetov, K. B.; Shuang, J.; Cava, R.; Taniguchi, T.; Watanabe, K.; Hone, J.; Dean, C. R.; Kim, P. Nat. Phys. 2016, 12, (4), 328-332.

18. Sahu, M. R.; Raychaudhuri, P.; Das, A. Phys. Rev. B 2016, 94, (23), 235451.

19. Likharev, K. K. Rev. Mod. Phys. 1979, 51, (1), 101-159.

20. Jeong, D.; Choi, J.-H.; Lee, G.-H.; Jo, S.; Doh, Y.-J.; Lee, H.-J. Phys. Rev. B 2011, 83, (9), 094503.

21. Choi, J.-H.; Lee, G.-H.; Park, S.; Jeong, D.; Lee, J.-O.; Sim, H. S.; Doh, Y.-J.; Lee, H.-J. Nat. Commun. 2013, 4, 2525.

22. Yabuki, N.; Moriya, R.; Arai, M.; Sata, Y.; Morikawa, S.; Masubuchi, S.; Machida, T. Nat. Commun. 2016, 7, 10616.

23. Lee, G.-H.; Kim, S.; Jhi, S.-H.; Lee, H.-J. Nat. Commun. 2015, 6.

24. Beenakker, C. W. J.; van Houten, H. Phys. Rev. Lett. 1991, 66, (23), 3056-3059.

25. Zazunov, A.; Shumeiko, V. S.; Bratus', E. N.; Lantz, J.; Wendin, G. Phys. Rev. Lett. 2003, 90, (8), 087003.

26. Bretheau, L.; Girit, C. O.; Pothier, H.; Esteve, D.; Urbina, C. Nature 2013, 499, (7458), $312-315$.

27. Pribiag, V. S.; BeukmanArjan, J. A.; Qu, F.; Cassidy, M. C.; Charpentier, C.;

Wegscheider, W.; Kouwenhoven, L. P. Nat. Nano. 2015, 10, (7), 593-597. 
28. Wang, L.; Meric, I.; Huang, P. Y.; Gao, Q.; Gao, Y.; Tran, H.; Taniguchi, T.; Watanabe, K.; Campos, L. M.; Muller, D. A.; Guo, J.; Kim, P.; Hone, J.; Shepard, K. L.; Dean, C. R. Science 2013, 342, (6158), 614-617.

29. Barone, A.; Johnson, W. J.; Vaglio, R. J. Appl. Phys. 1975, 46, (8), 3628-3632.

30. Schwidtal, K.; Finnegan, R. D. J. Appl. Phys. 1969, 40, (5), 2123-2127.

31. Barone, A.; Paternò, G., Physics and Applications of the Josephson Effect, John Wiley \& Sons: 2005; pp 96-120.

32. Elias, G.; Daniel, C.; Xi, X. X.; Ke, C. Supercond. Sci. Technol. 2014, 27, (6), 065015.

33. Tinkham, M., Introduction to Superconductivity: Second Edition. Dover Publications: 2004.

34. Klapwijk, T. M.; Blonder, G. E.; Tinkham, M. Physica $B+C$ 1982, 109, 1657-1664.

35. Morris, R. C.; Coleman, R. V. Phys. Lett. A 1973, 43, (1), 11-12.

36. Yokoya, T.; Kiss, T.; Chainani, A.; Shin, S.; Nohara, M.; Takagi, H. Science 2001, 294, (5551), 2518-2520.

37. Kulik, I. O.; Omel'yanchuk, A. N. Sov. J. Low Temp. Phys. 1977, 3, 459-462.

38. Nikolić, B. K.; Freericks, J. K.; Miller, P. Phys. Rev. B 2001, 64, (21), 212507.

39. Haberkorn, W.; Knauer, H.; Richter, J. phys. status solidi A 1978, 47, (2), K161-K164.

40. Beenakker, C. W. J. Phys. Rev. Lett. 1991, 67, (27), 3836-3839.

41. Kulik, I. O.; Omel'yanchuk, A. N. JETP Lett. 1975, 21, 96. 


\section{Strong proximity Josephson coupling in vertically}

stacked $\mathrm{NbSe}_{2}-$ graphene- $\mathrm{NbSe}_{2}$ van der Waals junctions

Minsoo Kim ${ }^{\dagger}$, Geon-Hyoung Park, Jongyoon Yi, Jae Hyeong Lee, Jinho Park, Hyunwoo Lee, Gil-Ho Lee, and Hu-Jong Lee*

Department of Physics, Pohang University of Science and Technology, Pohang 37673, Republic of Korea 


\section{Preparation of $\mathrm{NbSe}_{2}$-graphene-NbSe 2 heterostructure}

(a)

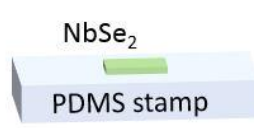

(b)

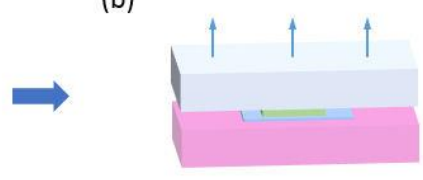

(e)

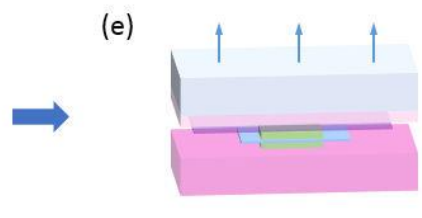

(g)

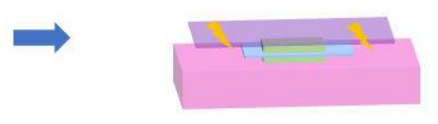

graphene
silicon substrate

(c)

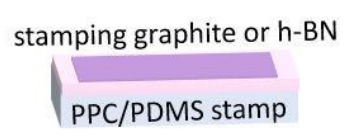

$\mathrm{NbSe}_{2}$

silicon substrate

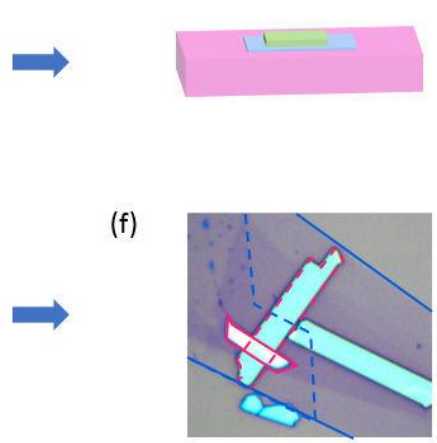

(h)

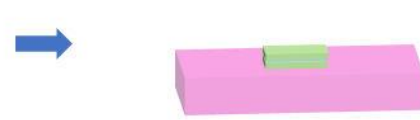

(d)

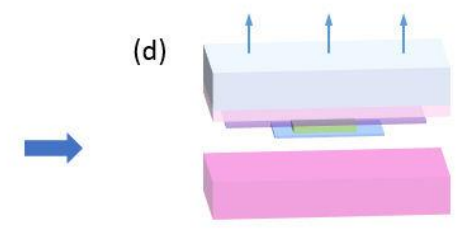

- stamping graphite

- - - graphene between top and bottom $\mathrm{NbSe}_{2}$

- top $\mathrm{NbSe}_{2}$

- - bottom $\mathrm{NbSe}_{2}$

Figure S1. (a) Exfoliation of thin flakes of $\mathrm{NbSe}_{2}$, graphene, and graphite (or hexagonal boron nitride h-BN) on each substrate. (b-c) Transfer of $\mathrm{NbSe}_{2}$ on top of the graphene. (d) Pick-up the heterostructure by graphite or h-BN. (e) Transfer of heterostructure onto the bottom $\mathrm{NbSe}_{2}$ on silicon substrate. Then lift only the PDMS stamp. (f) Optical microscope image of $\mathrm{NbSe}_{2}-$ graphene $-\mathrm{NbSe}_{2}$ heterostructure. Stamping graphite / graphene between top and bottom $\mathrm{NbSe}_{2}$ / top $\mathrm{NbSe}_{2}$ / bottom $\mathrm{NbSe}_{2}$ are outlined by solid blue / dashed blue / solid black / dashed black lines. (g) Selective plasma etching. (h) Preparation of $\mathrm{NbSe}_{2}-$ graphene $-\mathrm{NbSe}_{2}$ heterostructure. This work should be done as fast as possible to prevent the oxidization of $\mathrm{NbSe}_{2}$ flake. (i) Schematic configuration of the device fabricated by the processes. 
Drop of the junction resistance due to a non-uniform current distribution in the junction

Here we show a quantitative analysis on the anomalous drop of the junction resistance $R_{\mathrm{JJ}}$ above the critical current $\left(I_{\mathrm{c}}^{\text {top }}{ }_{, \mathrm{NbSe} 2}=1.5 \mathrm{~mA}\right)$ of top $\mathrm{NbSe}_{2}$ electrodes, while the bottom $\mathrm{NbSe}_{2}$ electrode stays superconducting. We used the commercial package COMSOL Multiphysics based on finite element analysis. This is the condition for the anomalous backward resistance drop for the bias above $I_{\mathrm{C}}{ }^{\text {top }}{ }_{\text {,NbSe2 }}$ in Fig. 1a. Qualitatively speaking, the anomalous drop of $R_{\mathrm{JJ}}$ is due to the non-uniform current flowing through the junction when any of electrodes (the top $\mathrm{NbSe}_{2}$ electrode in this case) has a finite square resistance comparable to or larger than the junction resistance itself. When resistivity of top electrode ( $\left.\rho^{\text {top }}{ }_{, \mathrm{NbSe} 2}\right)$ is negligible $\left(10^{-10} \Omega \mathrm{m}\right.$; simulating the superconducting state), the top $\mathrm{NbSe}_{2}$ electrode forms an equipotential layer, representing a uniform current flow as shown in Fig. $\mathrm{S} 2$. In this case, $R_{\mathrm{JJ}}$ would be measured correctly as $0.59 \Omega$. As resistivity of the top electrode becomes larger as for $I$ becomes slightly above $I_{\mathrm{c}}{ }^{\text {top }}{ }_{\text {NbSe2}}$, more current flows through the junction edge closer to the source. This results in the decrease of $R_{\mathrm{JJ}}$ approaching zero resistance. In the simulation, the resistivity of bottom $\mathrm{NbSe}_{2}$ electrode $\left(\rho^{\text {bottom }}{ }_{\text {,NbSe2}}\right)$ was set as small as $10^{-14} \Omega \mathrm{m}$ to represent its superconducting state. If the square resistance of the bottom electrode also becomes larger than the junction resistance the current distribution in the junction becomes more severely non-uniform, which results in even negative junction resistance in the cross-junction measurement configuration.

Figure $\mathrm{S} 3$ shows the simulated $R_{\mathrm{JJ}}$ as a function of the resistivity of the top $\mathrm{NbSe}_{2}$ electrode. Actual value of $\rho^{\text {top }}{ }_{\text {NbSe2 }} \sim 6 \times 10^{-8} \Omega \mathrm{m}$ (represented by red arrow in Fig. S3) was estimated using the normal-state resistance of top $\mathrm{NbSe}_{2}$ in the inset of Fig. 2a of the main text. Simulated value of $R_{\mathrm{JJ}} \sim 0.3 \Omega$ well matches with the observed resistance $(0.30 \Omega)$ for the bias current just above $I_{\mathrm{c}}{ }^{\text {top }}{ }_{\text {NbSe2}}$. 
Resistivity of bottom $\mathrm{NbSe}_{2}$ : $1 \mathrm{E}-14 \Omega \mathrm{m}$

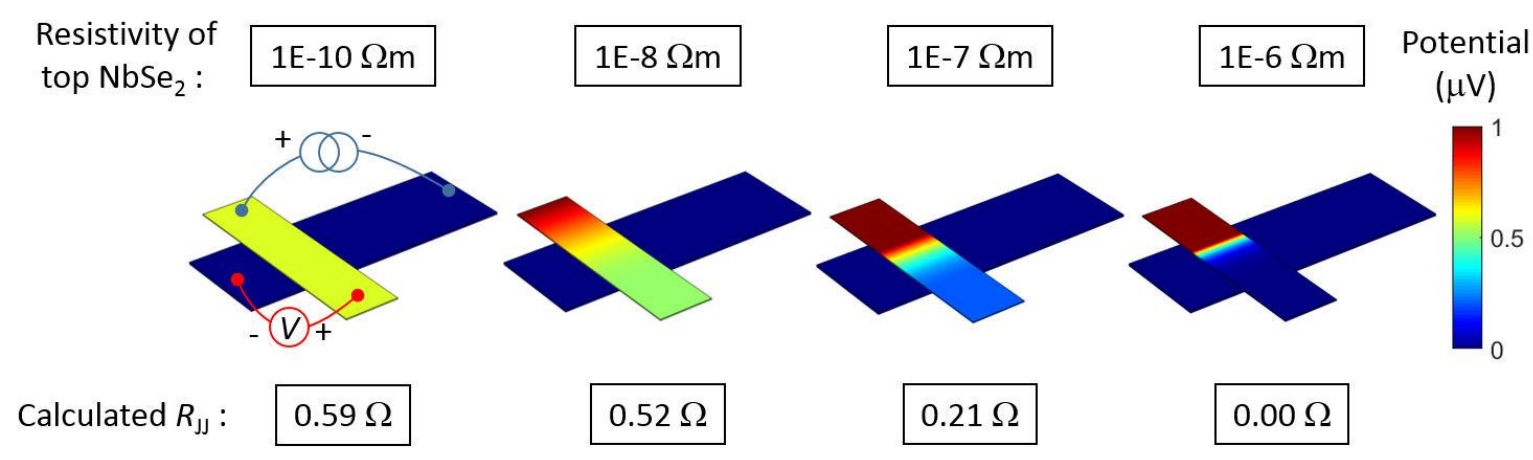

Figure S2. Potential map of the cross junction with the bias current of $1 \mu \mathrm{A}$ for different resistivity of $\mathrm{NbSe}_{2}$ electrodes.

(a)

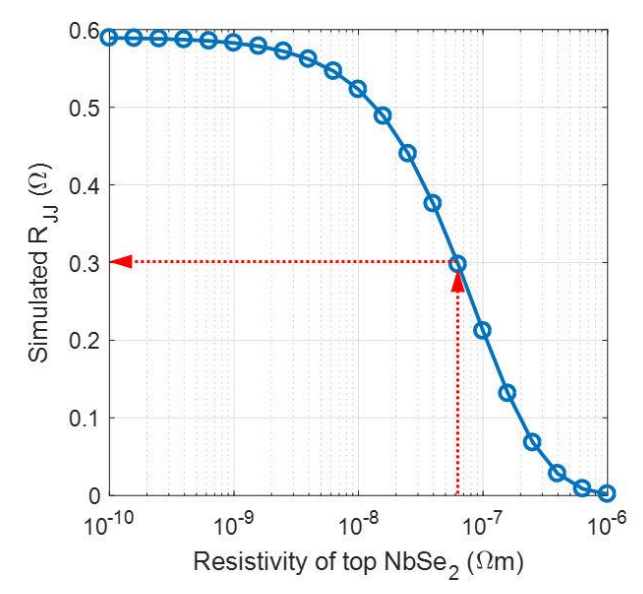

(b)
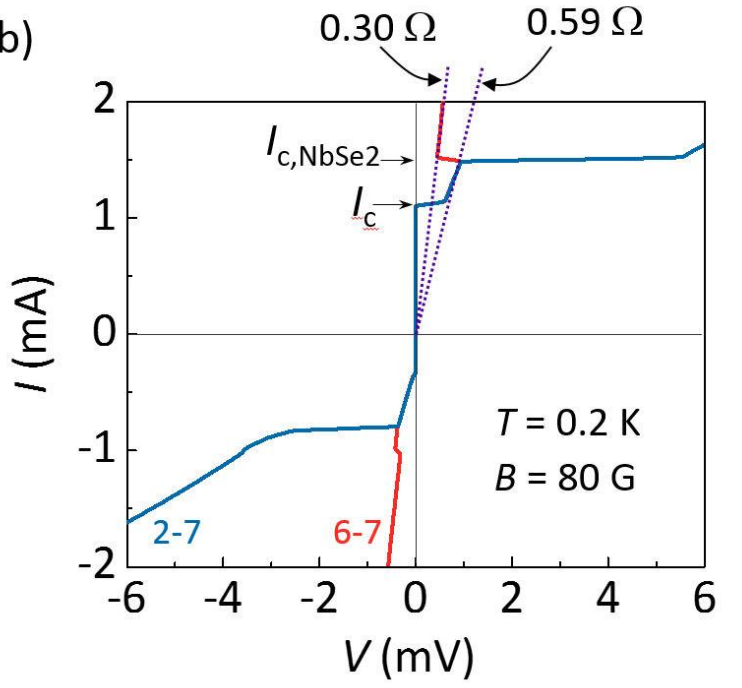

Figure S3. (a) Simulated junction resistance $\left(R_{\mathrm{JJ}}\right)$ as a function of the resistivity of the top $\mathrm{NbSe}_{2}$ electrode. Red arrows represent the case of the device in the main text. (b) The calculated junction resistance is in good agreement with the observed values near the anomalous jump. 


\section{Fraunhofer pattern of critical current at various temperature}
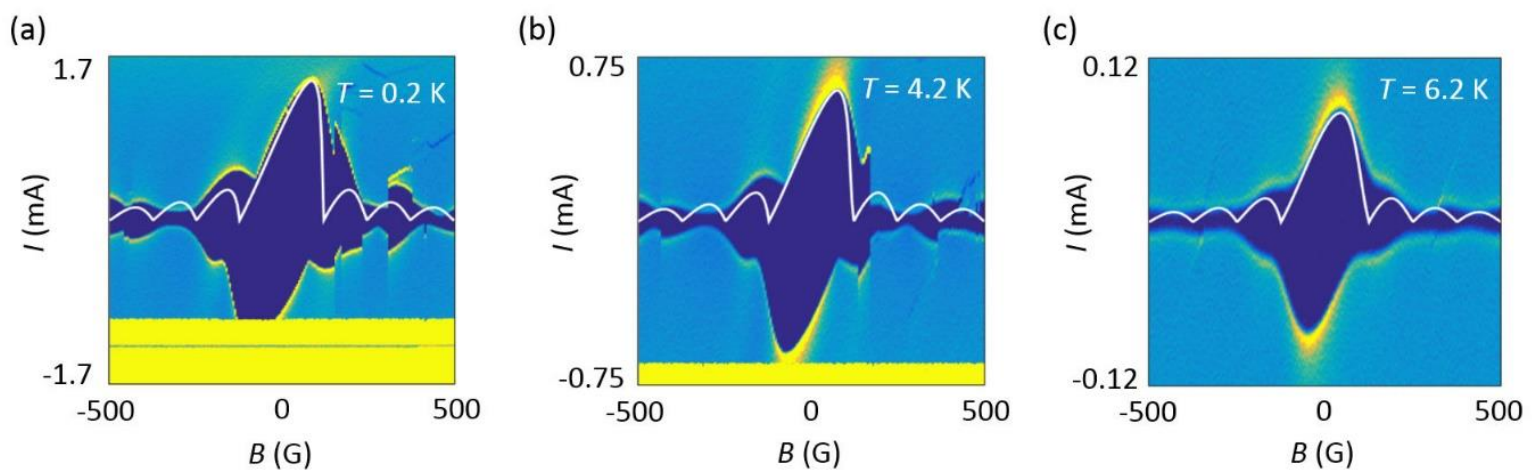

Figure S4. Fraunhofer pattern of critical current as a function of $B$-field measured at $T$ of (a) $0.2 \mathrm{~K}$, (b) $4.2 \mathrm{~K}$, and (c) $6.2 \mathrm{~K}$. Theoretical fitting in the consideration of self-field effect of uniform current density profile is overdrawn as white solid line. As temperature goes up, skewness in Fraunhofer pattern of $I_{\mathrm{c}}$ decreases due to the decrease in critical current density of Josephson junction. Maximum of $I_{\mathrm{c}}$ were at $B=80,80,30 \mathrm{G}$ each. 
Multiple Andreev reflection measured at temperature of $0.2 \mathrm{~K}$

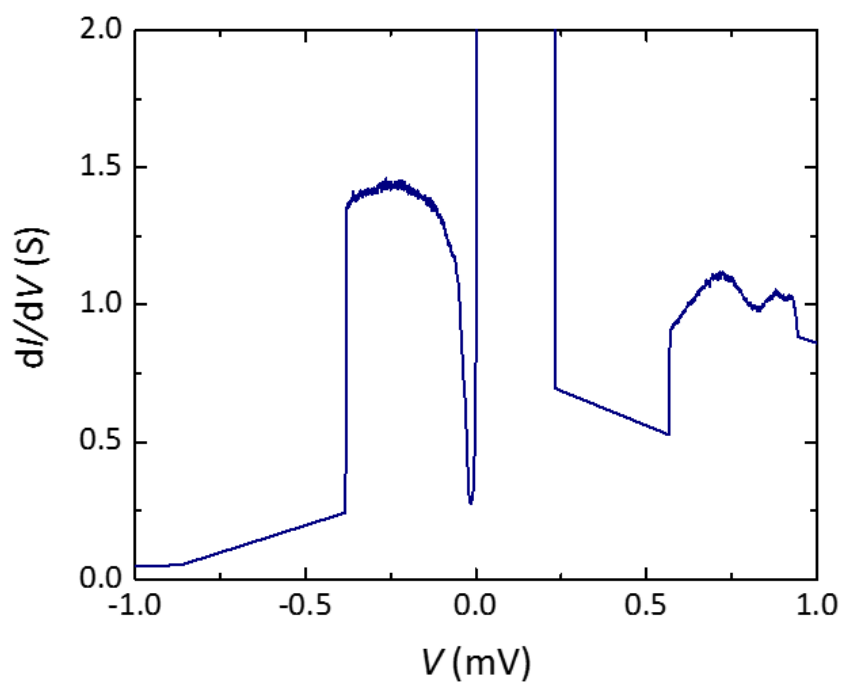

Figure S5. Multiple Andreev reflection (MAR) measured at $T=0.2 \mathrm{~K}$. MARs were barely visible at $V<0 \mathrm{mV}$. 
Measurements on other device - monolayer graphene, oxidized interface

(a)

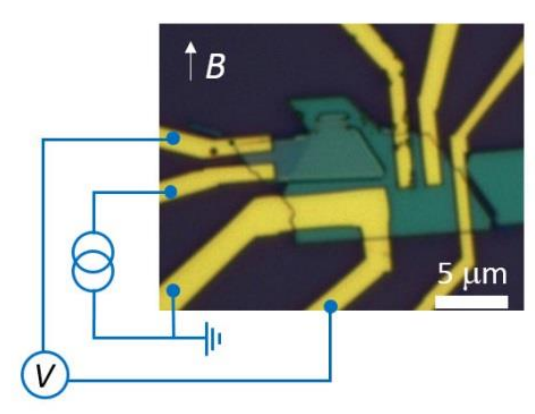

(b)

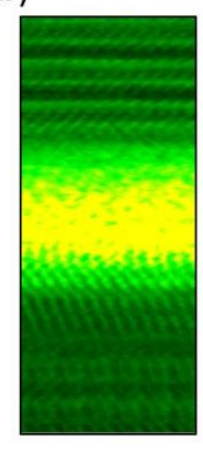

(c)

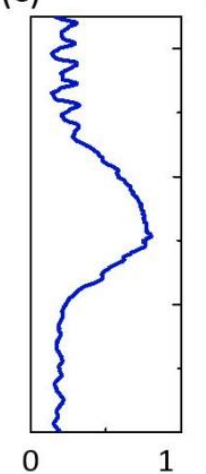

(d)

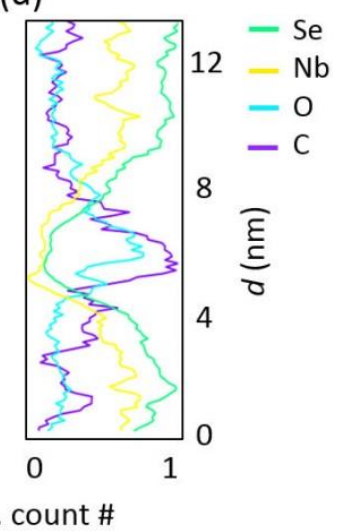

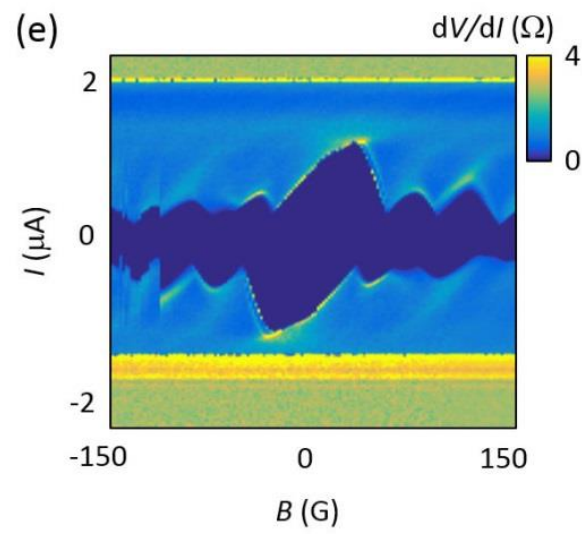

(f)

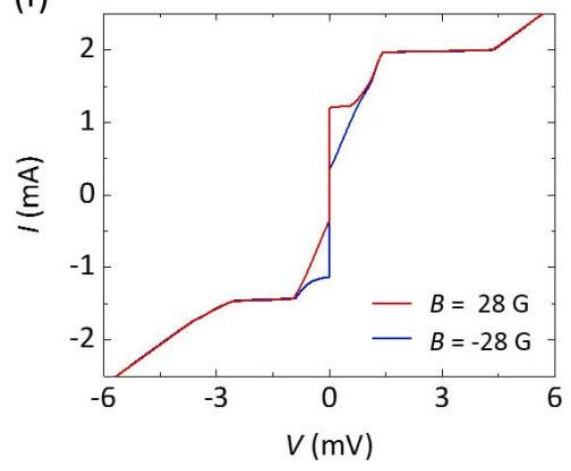

(h)
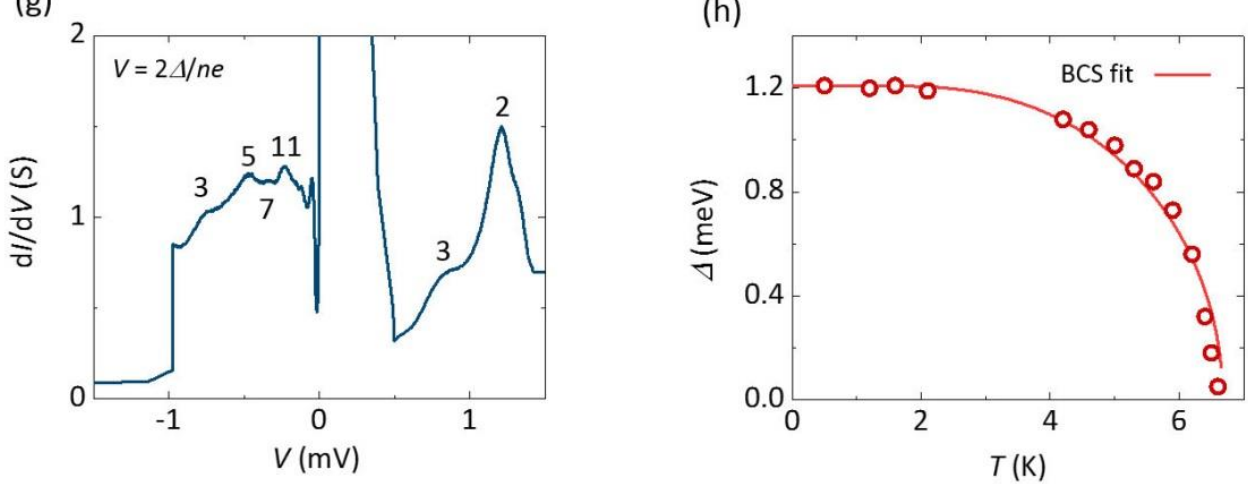

(i)

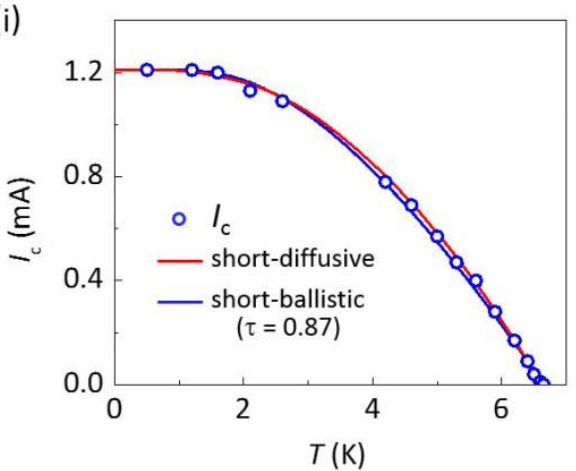


Figure S6. (a) Optical microscope image of the device with a measurements configuration. (b) Scanning transmission electron microscope (STEM) image of the junction vertical crosssection. (c) A slice profile of the STEM image and (d) corresponding energy dispersive spectroscopy data. Few-nm-thick oxidization at the interface between top-NbSe2 and graphene is visible. (e) Fraunhofer modulation of critical current as a function of $B$-field measured at $T=0.4 \mathrm{~K}$. (f) $I-V$ characteristic curves measured at $B=-28$ and $28 \mathrm{G}$ each, where maximums of critical current and retrapping current were obtained. (g) Multiple Andreev reflection (MAR) measured at $T=0.4 \mathrm{~K}$, where $\mathrm{d} I / \mathrm{d} V$ peaks satisfying the MAR relation $V=2 \Delta / n e(\mathrm{n}=2,3,5,7,11)$. (h) Temperature dependence of induced superconducting gap energy $\Delta$, best fitted to Bardden-Cooper-Schrieffer (BCS) theory. $\Delta$ and $T_{\mathrm{c}}$ of Josephson junction were $1.21 \mathrm{meV}$ and $6.65 \mathrm{~K}$. (i) Temperature dependence of critical current fitted to theoretical short-diffusive and short-ballistic model, where transparency $\tau$ was obtained to $0.87( \pm 0.03)$. Here, junction $T_{\mathrm{c}}=6.65 \mathrm{~K}$ was used for the fitting. The measured data of $I_{\mathrm{c}}$ as a function of $T$ are well fitted to both theories. Oxidized interface between different $\mathrm{NbSe}_{2}$ layer makes the junction transparency low. 


\section{Measurements on other device - tri-layer graphene}

(a)

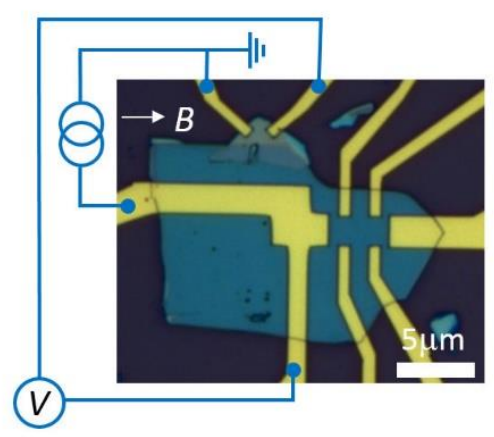

(b)

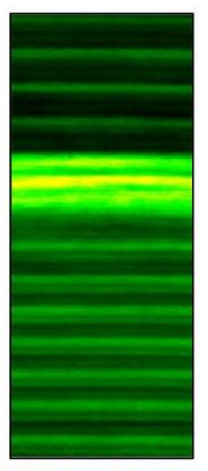

(c)

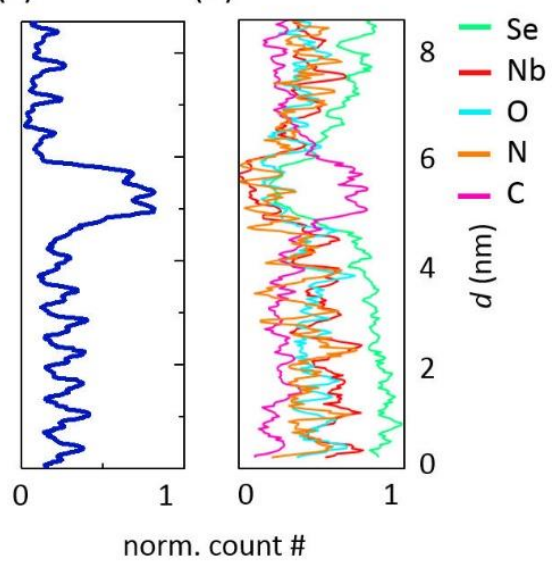

(e)

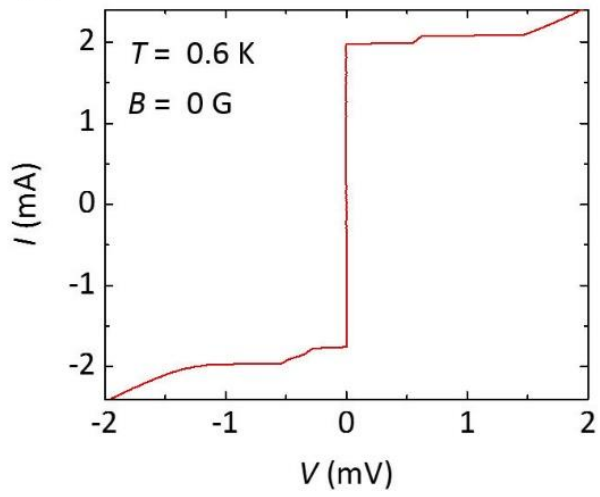

(f)

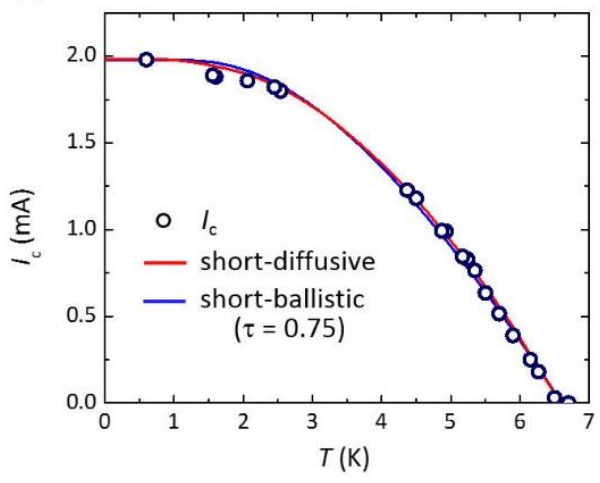

Figure S7. (a) Optical microscope image of the device with a measurements configuration.

Tri-layer graphene is used as a normal spacer between two $\mathrm{NbSe}_{2}$. (b) STEM image of the vertical cross-section of the junction. (c) Slice-cut of the STEM image and (d) EDS showing no oxidization at the interface of the junction (e) $I-V$ characteristic curves measured at $B=0 \mathrm{G}$. This device showed negligible self-field effect. Due to the short range of the $I-V$ curve, we could not get the relevant MAR in this device. (f) Temperature dependence of critical current fitted to theoretical short-diffusive and short-ballistic model, where transparency $\tau$ was estimated to $0.85( \pm 0.05)$. Here junction $T_{\mathrm{c}}=6.60 \mathrm{~K}$, superconducting gap energy of $1.2 \mathrm{meV}$ was used for the fitting. It is difficult to distinguish whether the junction is ballistic or diffusive by comparing the temperature dependence of $I_{\mathrm{c}}$. Transport through tri-layer graphene makes the junction less transparent. 